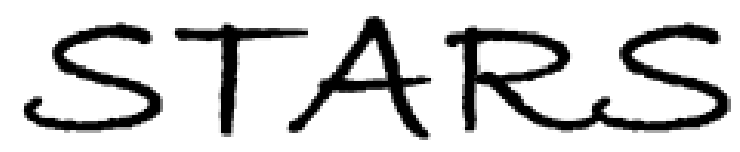

University of Central Florida

STARS

$1-1-2009$

\title{
Linear and nonlinear optical characterizations of a monomeric symmetric squaraine-based dye in solution
}

\author{
Carlo Toro \\ University of Central Florida \\ Leonardo De Boni \\ University of Central Florida \\ Sheng Yao \\ University of Central Florida \\ James P. Ritchie \\ University of Central Florida \\ Artëm E. Masunov \\ University of Central Florida \\ Find similar works at: https://stars.library.ucf.edu/facultybib2000 \\ University of Central Florida Libraries http://library.ucf.edu \\ See next page for additional authors \\ This Article is brought to you for free and open access by the Faculty Bibliography at STARS. It has been accepted for \\ inclusion in Faculty Bibliography 2000s by an authorized administrator of STARS. For more information, please \\ contactSTARS@ucf.edu.
}

\section{Recommended Citation}

Toro, Carlo; De Boni, Leonardo; Yao, Sheng; Ritchie, James P.; Masunov, Artëm E.; Belfield, Kevin D.; and Hernandez, Florencio E., "Linear and nonlinear optical characterizations of a monomeric symmetric squaraine-based dye in solution" (2009). Faculty Bibliography 2000s. 2234.

https://stars.library.ucf.edu/facultybib2000/2234

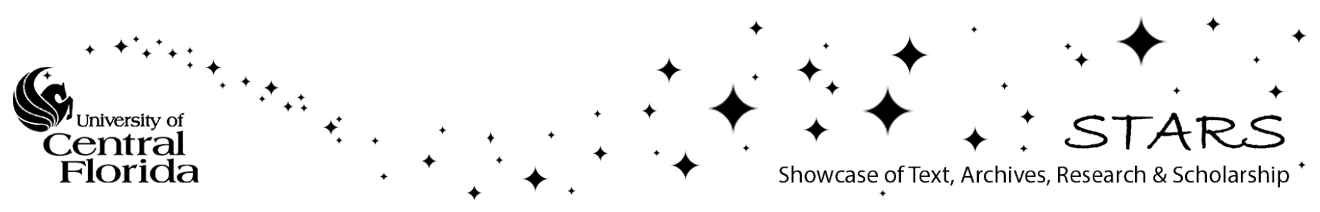




\section{Authors}

Carlo Toro, Leonardo De Boni, Sheng Yao, James P. Ritchie, Artëm E. Masunov, Kevin D. Belfield, and Florencio E. Hernandez 


\section{Linear and nonlinear optical characterizations of a monomeric symmetric squaraine-based dye in solution}

Cite as: J. Chem. Phys. 130, 214504 (2009); https://doi.org/10.1063/1.3147011

Submitted: 06 March 2009 . Accepted: 09 May 2009. Published Online: 02 June 2009

Carlo Toro, Leonardo De Boni, Sheng Yao, James P. Ritchie, Artëm E. Masunov, Kevin D. Belfield, and Florencio E. Hernandez

\section{ARTICLES YOU MAY BE INTERESTED IN}

Density-functional thermochemistry. III. The role of exact exchange

The Journal of Chemical Physics 98, 5648 (1993); https://doi.org/10.1063/1.464913

High efficiency organic photovoltaic cells based on a vapor deposited squaraine donor Applied Physics Letters 94, 233304 (2009); https://doi.org/10.1063/1.3152011
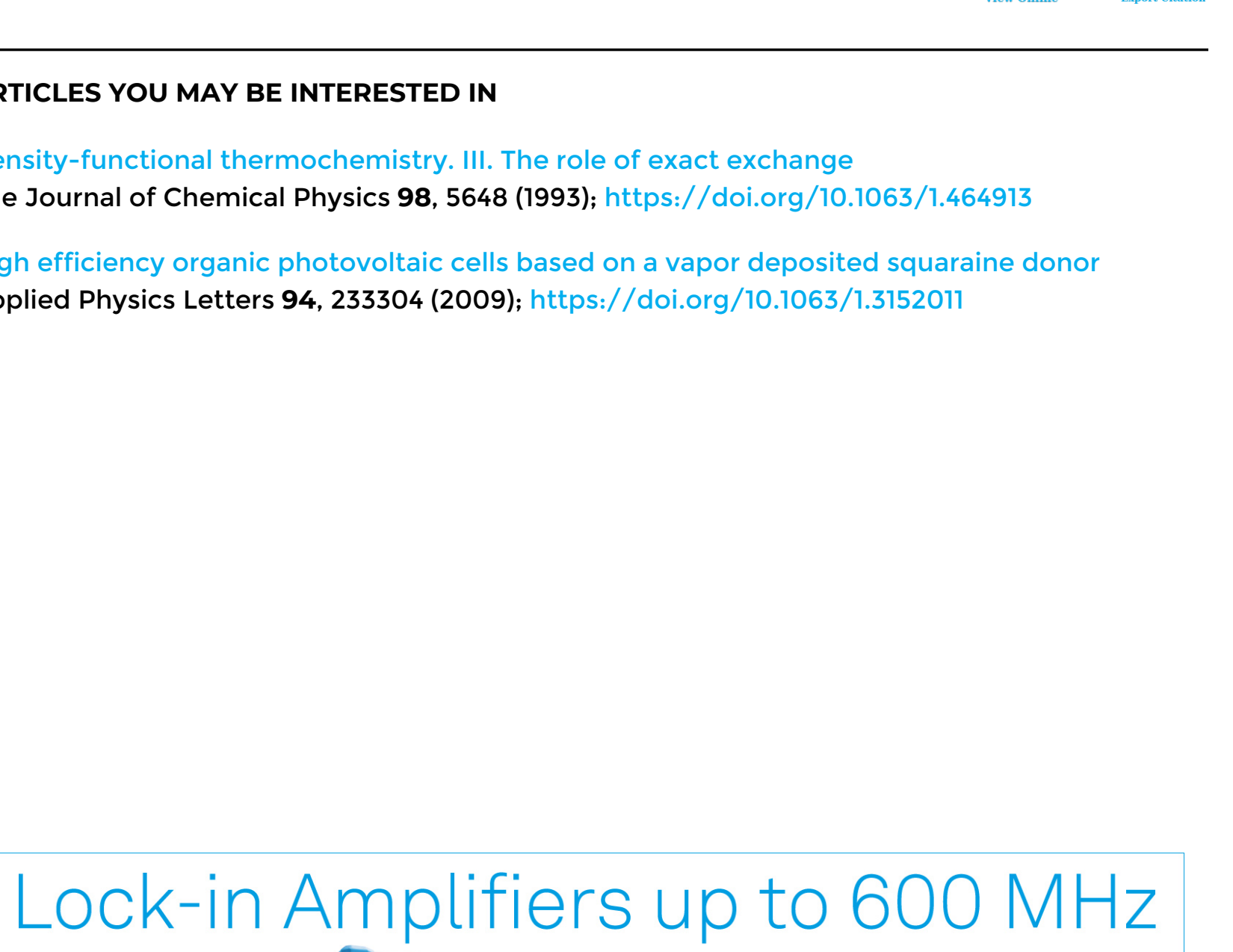

starting at $\$ 6,210$

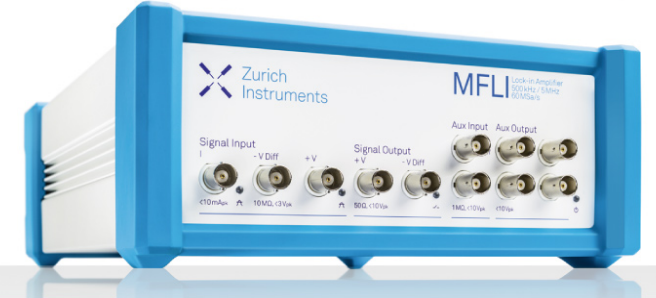

J. Chem. Phys. 130, 214504 (2009); https://doi.org/10.1063/1.3147011

(c) 2009 American Institute of Physics. 


\title{
Linear and nonlinear optical characterizations of a monomeric symmetric squaraine-based dye in solution
}

\author{
Carlo Toro, ${ }^{1}$ Leonardo De Boni, ${ }^{1}$ Sheng Yao, ${ }^{1}$ James P. Ritchie, ${ }^{2}$ Artëm E. Masunov, ${ }^{1,2,3}$ \\ Kevin D. Belfield, ${ }^{1, a)}$ and Florencio E. Hernandez ${ }^{1, a), b)}$ \\ ${ }^{1}$ Department of Chemistry, University of Central Florida, P.O. Box 162366, \\ Orlando, Florida 32816-2366, USA \\ ${ }^{2}$ NanoScience Technology Center, P.O. Box 162366, Orlando, Florida 32826, USA \\ ${ }^{3}$ Department of Physics, UCF, P.O. Box 162366, Orlando, Florida 32816-2366, USA
}

(Received 6 March 2009; accepted 9 May 2009; published online 2 June 2009)

\begin{abstract}
The photophysical properties of a symmetric squaryllium dye, namely, 2,4-bis[4-( $N, N$-dibutylamino)-2-hydroxyphenyl] squaraine (SQ), in its monomer form in acetone solution, have been thoroughly studied by means of one-photon absorption (1PA) and two-photon absorption (2PA), excitation anisotropy, fluorescence emission, fluorescence quantum yield, and excited state absorption. The results show that there is a strong one-photon allowed absorption band in the near IR region associated with intramolecular charge transfer. Higher one-photon allowed and forbidden singlet excited states were also revealed by absorption and excitation anisotropy. A relatively high fluorescence quantum yield (0.44) was measured for this dye. The nonlinear optical characterization of SQ in solution confirms the ability of squaraine dyes to be used as good two-photon absorbers. Additionally, it was found that this dye presents both saturable and reverse saturable absorption effects. Density functional theory calculations of the 1PA and 2PA electronic spectra of SQ were carried out to support the experimental data. A detailed analysis of the symmetry and energy of the orbitals involved in the lowest five electronic transitions is presented and discussed in relation to the behavior observed experimentally. (C) 2009 American Institute of Physics.
\end{abstract}

[DOI: $10.1063 / 1.3147011]$

\section{INTRODUCTION}

For several decades, organic-based materials have been sought out as potential candidates to develop electronic circuitry at the nanometer scale. The vast amount of information gathered over the years has led to a rational design of efficient synthetic strategies that easily allows one to tune the optical, electronic, and mechanical properties of most of these molecular systems at will. The ability to control the response of materials at the molecular level has had a tremendous impact in terms of applications, especially in key areas of optoelectronics devices, ${ }^{1}$ medicine, ${ }^{2}$ and nanotechnology. ${ }^{3,4}$ Nonetheless, the quest for more efficient and cost-effective materials is still an ongoing challenge. Therefore, some of the voids in this field that still remain to be covered call for systematic studies of the optical properties of organic materials and their structure-property relationship.

Among many of the already established organic archetypes for numerous applications, squaraine dyes represent one of the most interesting families to be studied. They are commonly prepared by reacting squaric acid, an electron deficient center, with strong electron-donating groups (nucleophile). ${ }^{5}$ The product is usually photostable because of the contribution of several resonance zwitterionic structures.

\footnotetext{
a) Also at CREOL/The College of Optics and Photonics, UCF.

b) Author to whom correspondence should be addressed. Electronic mail: florenzi@mail.ucf.edu.
}

Squaraine dyes adopt in general the form of donor-acceptordonor (D-A-D) system which tends to increase the electron delocalization through the whole structure of the molecule. This extended electron delocalization red shifts the main absorption band of squaraine dyes to the visible region of the spectrum. Commonly, the nature of the transition to the first singlet excited state in these molecules is associated with a strong intramolecular charge-transfer character and high molar extinction coefficients $\left(>10^{5} \mathrm{M}^{-1} \mathrm{~cm}^{-1}\right)$. Recently, Ajayaghosh and co-workers ${ }^{5,6}$ reviewed the versatile chemistry of squaraine dyes and highlighted the potential use of these compounds in fundamental and technological applications, for example, as optical switches, ${ }^{7}$ chemosensors, ${ }^{8}$ photodynamic therapy agents, ${ }^{9}$ near infrared fluorescent probes, ${ }^{10}$ and second-harmonic generation in Langmuir-Blodgett films. ${ }^{11}$

With regard to the linear and nonlinear optical properties of this type of molecular entities, some studies dealing with the excited state absorption and their promising ability to be used as efficient two-photon absorbers with high nonlinear absorption cross sections have been reported. ${ }^{12-14}$ Additionally, it has been shown that molecular aggregation plays a fundamental role in the chemical, electronic, and optical behaviors of these systems. ${ }^{15-17}$ Therefore, excitons, solutesolvent, and aggregate-solvent interactions are some of the key elements to consider when establishing a reliable structure-property relationship in squaraine dyes. Recently, in our group we have reported a tremendous enhancement of the multiphoton absorption of a cyanine derivative when 
forming $J$-aggregates in solution. ${ }^{18}$ Our previous research has led us to undertake a systematic study of the linear and nonlinear photophysical properties of various organic molecules in their monomeric and aggregated state.

Bearing in mind that squaraine dyes are among the most promising organic materials for photonics applications, herein we report the study of the linear and nonlinear optical properties of a symmetric squaraine dye in solution. With the aim of supporting the experimental results, theoretical calculations were carried out within the time-dependent density functional theory (TDDFT) and $\triangle \mathrm{SCF}$ framework, including solvent effects.

\section{MATERIAL AND METHODS}

2,4-bis[4-( $N, N$-dibutylamino)-2-hydroxyphenyl] squaraine (SQ) was synthesized in our group by standard procedures $^{19}$ and dissolved in acetone (Sigma-Aldrich). The linear absorption spectrum [one-photon absorption (1PA)] was collected with a single-beam spectrometer (Agilent 8453) using a $1 \mathrm{~mm}$ quartz cell (solvent and cell contributions were subtracted). The fluorescence spectrum was obtained by exciting the sample at $600 \mathrm{~nm}$ with an optical parametric generator (OPG) pumped by the third harmonic of a Nd:yttrium aluminum garnet (YAG) laser [full width at half maximum (FWHM) (=25 ps)] working at $10 \mathrm{~Hz}$ repetition rate. The fluorescence signal was collected through a multimode optical fiber coupled to an Andor Shamrock sr303 i spectrograph equipped with a DH720-18F-03 ICCD camera as detector. Linear optical effects were measured on SQ/acetone solutions at a concentration of $\sim 9 \times 10^{-5} M$. Fluorescence quantum yield, $\phi_{f}$, and excitation anisotropy were measured with a PTI Quanta Master spectrofluorimeter (model QM-3/2005) equipped with a $75 \mathrm{~W}$ xenon lamp and two QuadraScopic monochromators (excitation and emission). Fluorescence quantum yield were determined using the standard method ${ }^{20}$ with cresyl violet in methanol as reference $\left(\phi_{\text {std }}=0.54\right)$. The excitation anisotropy of SQ was measured in a $9 \times 10^{-5} M$ poly-THF (tetrahydrofuran) solution.

The excited state absorption and two-photon absorption (2PA) spectra were measured using the well-known open aperture Z-scan method. ${ }^{21}$ The excitation source for these experiments was the OPG-Nd:YAG laser described before. The Gaussian beam profile was improved by means of a spatial filter before the Z-scan setup. Two silicon detectors were used to measure the normalized transmittance. Nonlinear absorption measurements of SQ in acetone solution were carried out at a concentration of $\sim 9 \times 10^{-4} \mathrm{M}$.

Pump-probe experiments were performed using a whitelight continuum (WLC) generated in de-ionized water with picosecond pulses from the Nd:YAG laser as probe beam. ${ }^{22}$ In order to avoid any significant excitation of SQ, the WLC was attenuated using neutral density filters. The pump beam, however, had high enough energy $(\approx 20 \mu \mathrm{J})$ to populate the excited state with a great portion of molecules. The pump wavelength was tuned to $600 \mathrm{~nm}$ with the intention of having a strong excitation of the first visible band in SQ. With the purpose of obtaining the spectroscopic information of the excited state, a delay line was used to find the zero delay (temporal overlapping) condition.

In order to further understand the electronic structure of SQ in an acetone solution, theoretical calculations of the first five singlet excited states were performed within the TDDFT framework. ${ }^{23}$ Solvent effects were taken into account using the polarizable continuum model (PCM). ${ }^{24}$ All calculations were carried out with the GAUSSIAN 03 program $^{25}$ using the hybrid functional B3LYP (Refs. 26 and 27) and the 6-31G ${ }^{* * *}$ basis set. With the aim of reducing computation time, and based on the fact that different alkyl substituents do not exert any effect on the electronic properties of squaraine dyes of the type $\mathrm{D}-\mathrm{A}-\mathrm{D},{ }^{28,29}$ the $n$-butyl groups on the nitrogen were replaced by methyl groups. Furthermore, four different structures of the same compound were studied: (a) A fully planar structure with the hydrogens of the hydroxyl group on position 2 of the phenyl ring pointing away from the carbonylic oxygens of the squaric moiety, and forming no hydrogen bonds (SQ-I). (b) A totally planar structure with the hydrogens of the hydroxyl groups interacting through intramolecular hydrogen bonding with the oxygens on the $\mathrm{C}-\mathrm{O}$ bond of the squaric center (SQ-II). (c) A structure in which the dihedral angle between the squaric center and one of the phenyl rings has been twisted by $10^{\circ}$ without losing intramolecular hydrogen bonding (SQ-III). Finally, (d) a structure with the dihedral angle between the squaric moiety and both phenyl rings twisted symmetrically and with opposite sign $\left(10^{\circ}\right.$ and $-10^{\circ}$ ) preserving intramolecular hydrogen bonding (SQIV). Structures SQ-I and SQ-II were fully optimized under $C_{2 h}$ symmetry restriction at B3LYP/6-31G ${ }^{* *}$ level of theory. Next, structures SQ-III and SQ-IV were obtained by unsymmetric and symmetric rigid twist, respectively, of the dihedral angle between the squaric center and the phenyl ring in SQ-II. The $10^{\circ}$ twist angle was arbitrarily chosen to simulate the optical and electronic properties of one of the infinity possibilities of rearrangement when the molecules undergo tumbling in solution. No further optimization of such structures was carried out. Unsymmetric twisting led to a structure with no symmetry $\left(C_{1}\right)$ while symmetric twisting produced a structure with $C_{i}$ symmetry. Hydrogen bonding effects on the last three structures were of particular interest since it has been demonstrated that this is a key feature in the behavior of squaraine dyes. ${ }^{30,31}$

The predictions of the 2PA were performed as described next. For a single linear polarized laser beam, the 2PA cross section, averaged over molecular orientations, can be expressed in cgs units as follows: ${ }^{32}$

$$
\sigma^{(2)}(\omega)=\frac{8 \pi^{3} \omega^{2}}{15 c^{2}} \sum_{i} \sum_{\beta}^{x, y, z} \sum_{\alpha}^{x, y, z}\left(M_{\alpha \alpha}^{i} M_{\beta \beta}^{i}{ }^{*}+2 M_{\alpha \beta}^{i} M_{\alpha \beta}^{i}{ }^{*}\right) g_{i}(2 \omega) \text {. }
$$

Here $\alpha, \beta=x, y, z$, and $M$ is the so-called 2PA matrix element, expressed as the sum over states (SOS) through transition dipoles between the excited states: 


$$
M_{\alpha \beta}^{i}=\frac{1}{2 \hbar} \sum_{k}\left[\frac{\left\langle i\left|\overline{\mu_{\alpha}}\right| k\right\rangle\left\langle k\left|\mu_{\beta}\right| 0\right\rangle}{\omega_{k 0}-\omega-i \Gamma_{k}}+\frac{\left\langle i\left|\overline{\mu_{\beta}}\right| k\right\rangle\left\langle k\left|\mu_{\alpha}\right| 0\right\rangle}{\omega_{k 0}-\omega-i \Gamma_{k}}\right] .
$$

In this expression, the summation runs over all the excited states that may play a role of an intermediate state in the 2PA mechanism. The transition dipole moments between the $i$ th and $k$ th excited states (permanent dipoles, when $i=k$ ) are

$$
\left\langle i\left|\overline{\mu_{\alpha}}\right| k\right\rangle=\left\langle i\left|\mu_{\alpha}\right| k\right\rangle-\left\langle 0\left|\mu_{\alpha}\right| 0\right\rangle \delta_{i k} .
$$

Expression (1) describes the transition rate of the excitation of the molecules. It is worth noticing that Eq. (1) differs by factor of 2 from the expression derived by Ohta and Kamada, ${ }^{33}$ which describes the dissipation rate of photons of the incident light. To predict a 2PA spectrum, one needs transition dipole moments between excited states and permanent dipole moments for infinite number of excited states. These values can be obtained only if one retains the quadratic terms in external field while solving the time-dependent equations. This quadratic approximation uses the states obtained in the linear response approximation as the basis and involves summation over infinite number of these states. This summation can also be replaced by linear equations as it is done in quadratic response DFT. ${ }^{34}$ Alternatively, one can carry out explicit summation over sufficient number of the lowest excited states. ${ }^{35}$ The state to state transition dipoles and permanent excited state dipoles can also be approximated using the a posteriori Tamm-Dancoff approximation (ATDA), and substituted in Eq. (3) to predict 2PA. ATDA was proposed recently and validated numerically against wave function theory results on the example of $\pi-\pi^{*}$ excited states of the linear polyenes. ${ }^{36}$ We implemented ATDA in a modified version of the GAUSSIAN 2003 code. We also used the selfconsistent field procedure to optimize the orbitals for KohnSham determinant corresponding to the leading configuration in the particular excited state. Since such determinant is an equal mix of the singlet and triplet spin states, the energy of the pure singlet was obtained using sum rule, as suggested by Han et al.: ${ }^{37} E_{S}=2 E_{L S}-E_{T}$, where $E_{L S}$ is the energy of excited singlet $\mathrm{KS}$ determinant, and $E_{T}$ is the energy of the triplet with the same orbital occupation from UB3LYP calculations. This procedure is known as $\triangle \mathrm{SCF}$, it produces the excitation energy corrected to the infinite order. Hence, the 1PA and 2PA spectra were calculated at the TD-B3LYP/6-31G** level of theory, and the excitation energies were corrected to their respective $\triangle \mathrm{SCF}$ values. The permanent and state to state transition diploes were obtained using ATDA, implemented in the locally modified version of GAUSSIAN 2003 code. The SOS expressions (1) and (3) were used with the total number of states equal to 32 .

\section{RESULTS AND DISCUSSION}

Figure 1 displays the normalized absorption (solid line) and emission (dashed line) spectra of SQ dissolved in acetone as well as its excitation anisotropy spectrum (the structure of this dye is shown as an inset). In general, SQ dyes are known to present a strong absorption band in the near infrared region with a high molar extinction coefficient $(\varepsilon)$, attributed to the $S_{0} \rightarrow S_{1}\left(\pi \rightarrow \pi^{*}\right)$ transition. In our particular

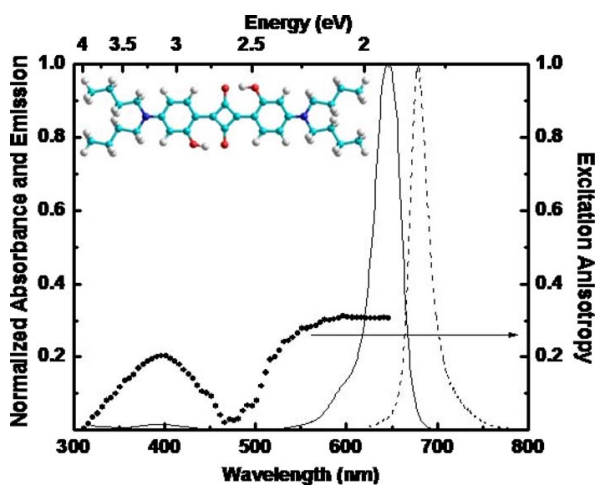

FIG. 1. (Color online) Normalized absorption (solid line) and emission (dashed line) spectra of 2,4-bis[4-( $N, N$-dibutylamino)-2-hydroxyphenyl] SQ in acetone solution. The excitation anisotropy (O) was measured in poly-THF. The inset shows the structure of SQ.

dye, this sharp transition occurs at an energy of $1.92 \mathrm{eV}$ $(645 \mathrm{~nm})$ with a value of $\varepsilon \approx 3 \times 10^{5} \mathrm{M}^{-1} \mathrm{~cm}^{-1}$. Similar absorption properties have been previously reported for this particular dye in a different solvent. ${ }^{16}$ Analysis of the absorption spectrum at higher energies reveals a region between 2.36 and $2.76 \mathrm{eV}$ with negligible absorption. Based on quantum mechanics selection rules and evaluating the symmetry of the molecule, this spectral region should correspond to a one-photon forbidden $S_{0} \rightarrow S_{2}$ transition. At higher energies, a second absorption peak, assigned to the $S_{0} \rightarrow S_{n}$ transition, was observed at approximately $3.14 \mathrm{eV}(395 \mathrm{~nm})$ and with an $\varepsilon \approx 5 \times 10^{3} \mathrm{M}^{-1} \mathrm{~cm}^{-1}$. These transitions have been corroborated by the analysis of the excitation anisotropy $\left(r_{0}\right)$ spectrum. This useful technique ${ }^{20}$ allows one to assign the spectral position of the different electronic transitions in a chromophore based on the estimation of the angle between the absorption and emission dipole moments. Theoretically, perfectly collinear dipoles give rise to the maximum value of excitation anisotropy (0.4) which corresponds to a transition $S_{0} \rightarrow S_{1}$. Changes in the slope of the excitation anisotropy profile are associated with transitions to higher excited states. Experimentally though, inhomogeneous broadening and overlapping between excited states play a key role in the energy distribution of the molecule and, therefore, the behavior observed in the $r_{0}$ spectrum should be interpreted carefully. In SQ, the constant value of approximately 0.3 on the excitation anisotropy, reached between 1.90 and $2.26 \mathrm{eV}$ $(650-550 \mathrm{~nm})$, can be attributed to a nearly pure transition from the ground state to the first singlet excited state. The main band in this spectral region observed in the linear absorption spectrum supports this result. A change in the slope in the region spanning from 2.26 to $2.70 \mathrm{eV}(550-460 \mathrm{~nm})$ suggests that the second excited state is being reached. However, the lack of a constant value throughout this energy range implies that this transition is not totally pure but rather overlapped with transitions to the first and other excited states. Finally, a spectral region between 2.70 and $3.18 \mathrm{eV}$ $(460-390 \mathrm{~nm})$ is clearly observed and ascribed to an $S_{0}$ $\rightarrow S_{n}$ transition.

To fully characterize the linear optical properties of this molecule, the fluorescence spectrum of SQ was also taken in acetone. The fluorescence emission peak was observed at 
TABLE I. First five singlet excited states of the four different configurations of SQ studied in this work. The values in parenthesis represent the contribution (percentage) of that particular transition to the overall transition.

\begin{tabular}{|c|c|c|c|c|c|}
\hline Structure & Transition & Molecular orbitals' contributions & $\begin{array}{c}E \\
(\mathrm{eV})\end{array}$ & Final state symmetry & $f$ \\
\hline \multirow[t]{5}{*}{ SQ-I } & $S_{0} \rightarrow S_{1}$ & $|\mathrm{H} \rightarrow \mathrm{L}\rangle(71.7)$ & 2.26 & $B_{u}$ & 1.649 \\
\hline & $S_{0} \rightarrow S_{2}$ & $|\mathrm{H}-1 \rightarrow \mathrm{L}\rangle(97.8)$ & 2.36 & $B_{g}$ & 0.000 \\
\hline & $S_{0} \rightarrow S_{3}$ & $|\mathrm{H}-3 \rightarrow \mathrm{L}\rangle(88.5)+|\mathrm{H}-5 \rightarrow \mathrm{L}\rangle(4.4)$ & 3.25 & $B_{u}$ & 0.081 \\
\hline & $S_{0} \rightarrow S_{4}$ & $|\mathrm{H}-4 \rightarrow \mathrm{L}\rangle(62.6)+|\mathrm{H}-2 \rightarrow \mathrm{L}\rangle(29.8)+|\mathrm{H} \rightarrow \mathrm{L}+1\rangle(2.2)$ & 3.25 & $A_{g}$ & 0.000 \\
\hline & $S_{0} \rightarrow S_{5}$ & $|\mathrm{H}-6 \rightarrow \mathrm{L}\rangle(97.1)$ & 3.33 & $A_{u}$ & 0.000 \\
\hline \multirow[t]{5}{*}{ SQ-II } & $S_{0} \rightarrow S_{1}$ & $|\mathrm{H} \rightarrow \mathrm{L}\rangle(71.9)$ & 2.30 & $B_{u}$ & 1.772 \\
\hline & $S_{0} \rightarrow S_{2}$ & $|\mathrm{H}-1 \rightarrow \mathrm{L}\rangle(66.3)+|\mathrm{H}-3 \rightarrow \mathrm{L}\rangle(25.9)$ & 3.00 & $B_{u}$ & 0.070 \\
\hline & $S_{0} \rightarrow S_{3}$ & $|\mathrm{H}-2 \rightarrow \mathrm{L}\rangle(91.5)$ & 3.00 & $A_{g}$ & 0.000 \\
\hline & $S_{0} \rightarrow S_{4}$ & $|\mathrm{H}-4 \rightarrow \mathrm{L}\rangle(98.3)$ & 3.15 & $B_{g}$ & 0.000 \\
\hline & $S_{0} \rightarrow S_{5}$ & $|\mathrm{H}-3 \rightarrow \mathrm{L}\rangle(62.6)+|\mathrm{H}-1 \rightarrow \mathrm{L}\rangle(20.0)+|\mathrm{H} \rightarrow \mathrm{L}+2\rangle(5.6)$ & 3.47 & $A_{g}$ & 0.000 \\
\hline \multirow[t]{5}{*}{ SQ-III } & $S_{0} \rightarrow S_{1}$ & $|\mathrm{H} \rightarrow \mathrm{L}\rangle(71.8)$ & 2.29 & $A$ & 1.748 \\
\hline & $S_{0} \rightarrow S_{2}$ & $\begin{aligned}|\mathrm{H}-2 \rightarrow \mathrm{L}\rangle(70.1)+\mid \mathrm{H}-4 & \rightarrow \mathrm{L}\rangle(4.6)+|\mathrm{H}-3 \rightarrow \mathrm{L}\rangle(8.3)+\mid \mathrm{H}-1 \\
& \rightarrow \mathrm{L}\rangle(9.4)\end{aligned}$ & 2.99 & $A$ & 0.063 \\
\hline & $S_{0} \rightarrow S_{3}$ & $\begin{array}{c}|\mathrm{H}-1 \rightarrow \mathrm{L}\rangle(51.5)+|\mathrm{H}-3 \rightarrow \mathrm{L}\rangle(22.6)+|\mathrm{H}-2 \rightarrow \mathrm{L}\rangle(17.9) \\
|\mathrm{H}-4 \rightarrow \mathrm{L}\rangle(86.2)+|\mathrm{H}-3 \rightarrow \mathrm{L}\rangle(2.3)+|\mathrm{H}-2 \rightarrow \mathrm{L}\rangle(3.6)+\mid \mathrm{H}-1\end{array}$ & 3.00 & $A$ & 0.016 \\
\hline & $S_{0} \rightarrow S_{4}$ & $\begin{aligned} & \rightarrow \mathrm{L}\rangle(4.9) \\
|\mathrm{H}-3 \rightarrow \mathrm{L}\rangle(55.8)+\mid \mathrm{H}-1 & \rightarrow \mathrm{L}\rangle(20.3)+|\mathrm{H}-4 \rightarrow \mathrm{L}\rangle(6.9)+\mid H \rightarrow \mathrm{L}\end{aligned}$ & 3.12 & $A$ & 0.004 \\
\hline & $S_{0} \rightarrow S_{5}$ & $+2\rangle(5.3)$ & 3.46 & $A$ & 0.000 \\
\hline \multirow[t]{5}{*}{ SQ-IV } & $S_{0} \rightarrow S_{1}$ & $|\mathrm{H} \rightarrow \mathrm{L}\rangle(72.1)$ & 2.30 & $A_{u}$ & 1.760 \\
\hline & $S_{0} \rightarrow S_{2}$ & $|\mathrm{H}-3 \rightarrow \mathrm{L}\rangle(47.0)+|\mathrm{H}-1 \rightarrow \mathrm{L}\rangle(36.7)+|\mathrm{H}-4 \rightarrow \mathrm{L}\rangle(10.8)$ & 2.98 & $A_{g}$ & 0.000 \\
\hline & $S_{0} \rightarrow S_{3}$ & $|\mathrm{H}-2 \rightarrow \mathrm{L}\rangle(91.6)$ & 3.01 & $A_{u}$ & 0.062 \\
\hline & $S_{0} \rightarrow S_{4}$ & $\begin{array}{c}|\mathrm{H}-4 \rightarrow \mathrm{L}\rangle(63.4)+|\mathrm{H}-1 \rightarrow \mathrm{L}\rangle(29.4) \\
|\mathrm{H}-3 \rightarrow \mathrm{L}\rangle(41.1)+|\mathrm{H}-4 \rightarrow \mathrm{L}\rangle(23.0)+|\mathrm{H}-1 \rightarrow \mathrm{L}\rangle(19.7)+\mid \mathrm{H} \rightarrow \mathrm{L}\end{array}$ & 3.08 & $A_{g}$ & 0.000 \\
\hline & $S_{0} \rightarrow S_{5}$ & $+2\rangle(5.0)$ & 3.47 & $A_{g}$ & 0.000 \\
\hline
\end{tabular}

$1.83 \mathrm{eV}(680 \mathrm{~nm})$ which corresponds to a Stokes' shift of $0.09 \mathrm{eV}$. The measured fluorescence quantum yield, $\phi_{f}$ $=0.44$, constitutes an indication of the potential applications of this dye in imaging and dye lasing, among others. In fact, lasing of this dye was observed at $678 \mathrm{~nm}$ with a FWHM of $7 \mathrm{~nm}$. On the other hand, the pump-probe experiments showed that the fluorescence lifetime $\left(\tau_{f}\right)$ of SQ in acetone is in the order of $3 \mathrm{~ns}$. Hence, assuming negligible population of the triplet excited state through intersystem crossing, ${ }^{38}$ these values allowed us to estimate the radiative and nonradiative decay rates, $\Gamma=\phi_{f} / \tau_{f}=1.47 \times 10^{8} \mathrm{~s}^{-1}$ and $k_{\mathrm{nr}}=(1$ $\left.-\phi_{f}\right) / \tau_{f}=1.87 \times 10^{8} \mathrm{~s}^{-1}$, respectively. The recovered values suggest that in SQ, internal conversion (vibrational relaxation) and energy dissipation through solute-solvent interaction is marginally the most likely relaxation mechanism to take place $\left(k_{\mathrm{nr}} / \Gamma=1.3\right)$.

Next, in Table I we summarize the results predicted for the different vertical transitions in each of the proposed structures. The contributions of the Kohn-Sham orbitals to each of the electronic transition are also presented, including the highest occupied molecular orbital (HOMO) referred to as $\mathrm{H}$ and the lowest unoccupied molecular orbital (LUMO) referred to as L, and other orbitals lying below and above. Overall, one can observe that even though solvent effects were included, the standard TD-DFT method does not accurately reproduce the experimental absorption maximum $\left(S_{0}\right.$ $\rightarrow S_{1}$ ) in any of the structures. The comparison between the calculated and the experimental values for the most stable molecular configuration SQ-II are presented on Table II, together with predicted 2PA cross sections. The large deviation $(0.37 \mathrm{eV})$ for the predicted first excitation energy for cyanine dyes is one of the known failures of TD-DFT formalism. Recently, Jacquemin et al. ${ }^{39}$ reported that even long-range corrected functionals do not improve this situation. However, $\Delta \mathrm{SCF}$ result is much closer to experiment for the state $S_{1}$, which is expected for a transition with a strong intramolecular charge-transfer character. In addition, it can be noticed that intramolecular hydrogen bonding (SQ-II, SQ-III, and

TABLE II. Comparison between calculated ( $E^{\mathrm{TD}-\mathrm{DFT}}$ and $\left.E^{\triangle \mathrm{SCF}}\right)$ and experimental $\left(E^{\text {exp }}\right)$ energies of the first five singlet states of SQ-II. The calculated 2PA cross-section maximum is presented as well.

\begin{tabular}{lccccccc}
\hline \hline State & Leading configuration & State symmetry & $\begin{array}{c}E^{\mathrm{TD}-\mathrm{DFT}} \\
(\mathrm{eV})\end{array}$ & $\begin{array}{c}E^{\Delta \mathrm{SCF}} \\
(\mathrm{eV})\end{array}$ & $\begin{array}{c}E^{\exp } \\
(\mathrm{eV})\end{array}$ & $f$ & $\begin{array}{c}\sigma^{2} \\
(\mathrm{GM})\end{array}$ \\
\hline$S_{1}$ & $|\mathrm{H} \rightarrow \mathrm{L}\rangle$ & $B_{u}$ & 2.30 & 1.77 & 1.91 & 1.77 & 0 \\
$S_{2}$ & $|\mathrm{H}-2 \rightarrow \mathrm{L}\rangle$ & $B_{u}$ & 3.00 & $\cdots$ & 3.14 & 0.07 & 0 \\
$S_{3}$ & $|\mathrm{H}-1 \rightarrow \mathrm{L}\rangle$ & $A_{g}$ & 3.00 & 2.89 & 2.90 & 0.00 & 785 \\
$S_{4}$ & $|\mathrm{H}-4 \rightarrow \mathrm{L}\rangle$ & $B_{g}$ & 3.15 & 3.18 & & 0.00 & 0 \\
$S_{5}$ & $|\mathrm{H}-3 \rightarrow \mathrm{L}\rangle$ & $A_{g}$ & 3.47 & $\cdots$ & 3.30 & 0.00 & 33400 \\
\hline \hline
\end{tabular}


SQ-IV) generates a slight hypsochromic shift of the first electronic transition. This effect has already been observed experimentally in other squaraine derivatives. ${ }^{31,38}$ Conversely, it can be noticed that the theoretical transitions calculated with the $\triangle \mathrm{SCF}$ method provide a value closer to the experimental one $(\sim 0.11 \mathrm{eV})$. In general, one might expect that due to the stochastic dynamics of the molecules in solution, all the structures studied along with some other molecular configurations should contribute to the observed experimental spectrum. However, the analysis of the symmetry of the final state, associated with each transition and its corresponding energy allows one to identify the most plausible structure. In terms of symmetry, it was recently stated by Ohira et al $^{40}$ that due to the fact that most SQ dyes present an inversion symmetry operation, the 2PA process (nonlinear absorption discussed below) observed experimentally must respect symmetry selection rules imposed by quantum mechanics, i.e., from a ground gerade $\left(A_{g}\right)$ state to an excited gerade $\left(A_{g}\right)$ state. This observation suggests that the unsymmetric SQ-III is one of the least feasible structures in solution. The fact that the first four excited states present finite oscillator strength on transitions not observed in the experimental linear absorption spectrum corroborates this statement. Then again, if we bear in mind that intramolecular hydrogen bonding plays a fundamental role in the stabilization of squaraine dyes in solution, then SQ-I becomes the next least probable candidate. Although the fourth excited state has the right symmetry, it appears at an energy that can be considered too high relative to the experimental. In contrast, SQ-II and SQ-IV have a third and a second excited state, respectively, with the appropriate symmetry to undergo a two-photon transition. As expected, the one-photon oscillator strength for such transitions is zero. In the case of SQII, the second and third excited states are degenerated, matching the behavior observed in the linear absorption and excitation anisotropy spectra. At the same time, the spectral position of the higher excited state (second absorption peak) is well reproduced within $4 \%$ of the experimental value. All these features suggest that SQ-II and SQ-IV might be the most likely conformations of SQ in solution. Yet, $\mathrm{x}$-ray crystallography data ${ }^{41}$ and theoretical calculations ${ }^{30}$ show evidence of a planar structure for this molecule, supporting SQ-II as the conformation with the highest probability to occur in solution. In this sense, Fig. 2 shows the energy diagram for SQ-II highlighting the one-photon and twophoton allowed and forbidden transitions based on the symmetry of the states involved in the transition. The red crosses represent transitions that are forbidden by quantum mechanic selection rules. Even though the energy gap between states has been approximated to symbolize the energy of each individual state, one has to keep in mind the vibrational broadening leading to the overlap between the states. Additionally, the most important Kohn-Sham orbitals of the same structure are depicted. It can be observed that these orbitals are highly symmetric and spread through the entire structure indicating that electron delocalization occurs from the donor groups to the center of the molecule. A similar electron distribution for these frontier orbitals was found for all the conformations studied.

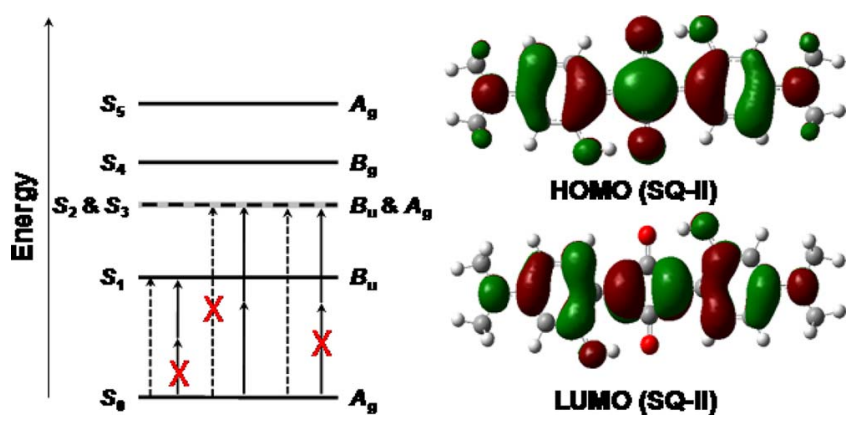

FIG. 2. (Color online) Energy diagram of the most probable conformation of SQ (SQ-II) calculated at TDDFT/PCM/B3LYP/6-31G** level of theory. Red crosses correspond to forbidden transitions based on quantum mechanics selection rules. The frontiers orbitals (HOMO and LUMO) for the same structure are also depicted.

With the idea of exploiting SQ dyes as materials with potential applications in nonlinear optics, measurements of excited state absorption and 2PA of SQ in solution were pursued in the same solvent. Figure 3 portrays the experimental one-photon (solid line), excited state (solid circles), and twophoton (solid squares) absorption cross-section spectra. For the particular case of the 2PA spectrum, the wavelengths plotted correspond to $\lambda_{\text {pump }} / 2$. The lines observed in the excited state and 2PA cross-section spectra were drawn to guide the reader and are not meant to be a fitting. To begin with, it can be noticed that this dye presents a negligible excited state absorption below $2.07 \mathrm{eV}$. This saturable absorption effect $\left(\sigma_{\text {ground }}>\sigma_{\text {excited }}\right)$ matches the same spectral region where the molecule presents its maximum 1PA probability. At higher energies, reverse saturable absorption $\left(\sigma_{\text {ground }}\right.$ $\left.<\sigma_{\text {excited }}\right)$ takes place within the spectral region in which the dye shows a vanishing 1PA cross section. The excited state absorption cross sections were determined following the rate equations analysis proposed for a three-level system. Alternatively, the 2PA spectrum was obtained by adjusting the individual Z-scan signatures using Sheik-Bahae's equations. ${ }^{21}$ The spectrum clearly obeys the selection rules for a symmetric molecule, i.e., it vanishes in the spectral region where only one-photon transitions are allowed (below $2.36 \mathrm{eV}$ ) and increases in the region where these are forbidden. The fact that $2 \mathrm{PA}$ is being achieved even within the second linear absorption peak confirms a strong overlap be-

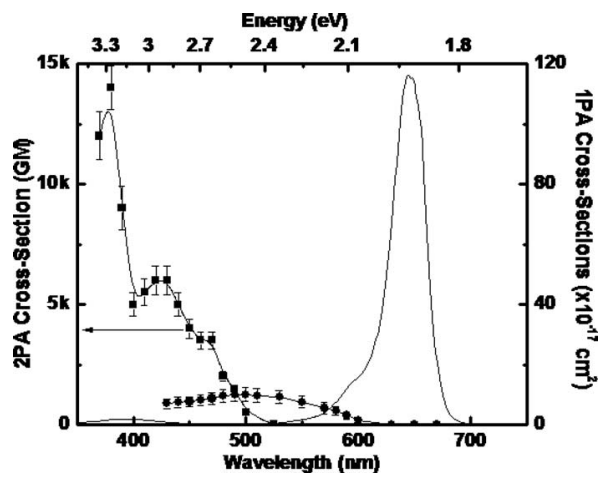

FIG. 3. Linear (solid line) and excited state ( ) absorption cross-section spectra of SQ in acetone. The 2PA cross-section spectrum ( $\mathbf{\square})$ is portrayed as well. 


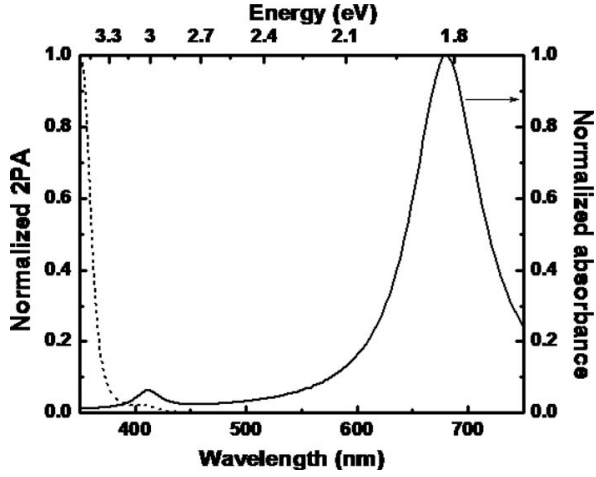

FIG. 4. Theoretical linear (solid line) and 2PA (dashed line) normalized absorption spectra of SQ in acetone, calculated using the combined $\mathrm{TD}-\mathrm{DFT} / \triangle \mathrm{SCF} / \mathrm{PCM}$ approach as described in Sec. II.

tween $S_{2}$ and $S_{3}$ as evidenced from the theoretical calculations and the excitation anisotropy. The relatively high 2PA cross sections obtained for energies greater than $3.10 \mathrm{eV}$ are due to near-resonance enhancement of the $S_{5}$ state absorption originating from the $S_{1}$ state. $^{40}$

In Fig. 4, we show the theoretical 1PA and 2PA normalized spectra calculated for $\mathbf{S Q - I I}$ using solvent effects. This calculation clearly reproduces the behavior observed experimentally. Our simulations suggest, as stated before, that the enhancement of the experimental 2PA signal is indeed occasioned by the proximity of the photon energy to the first excited state energy combined with a transition to a higher 2PA allowed state. The maximum cross section observed for the enhancement effect and the 2PA band, centered at $410 \mathrm{~nm}$, is $\sim 33000$ and $\sim 800 \mathrm{GM}$, respectively. The differences between these values and the experimental ones are attributed to the excited state absorption during the picosecond excitation pulse time.

\section{CONCLUSION}

Based on the potential of SQ derivatives to be used as key materials in the design of photonics and optoelectronics devices, we have explored the linear and nonlinear optical properties of a symmetric squaryllium dye, 2,4-bis [4- $(N, N$ dibutylamino)-2-hydroxyphenyl] SQ, in acetone solutions. Particularly, this study focused on the characterization of such a dye in its monomer form and interpretation of its electronic structure using the DFT. This work represents the first one in a series of studies aiming to exploit and understand the aggregation properties of these and some other organic moieties for potential applications in nonlinear optics. It was found that this particular molecule present welldefined absorption regions and a relatively high radiative efficiency. SQ has strong 2PA cross sections making it a good candidate for applications in photonics. Moreover, saturable and reverse saturable absorption effects were observed in different spectral regions.

\section{ACKNOWLEDGMENTS}

This work was supported by the National Science Foundation (Grant No. CHE-0832622).
${ }^{1}$ Y. Shirota, J. Mater. Chem. 10, 1 (2000).

${ }^{2}$ R. Langer and D. A. Tirrell, Nature (London) 428, 487 (2004).

${ }^{3}$ M. Schiek, F. Balzer, K. Al-Shamery, J. R. Brewer, A. Lutzen, and H.-G. Rubahn, Small 4, 176 (2008).

${ }^{4}$ D. A. Bonnell, J. Vac. Sci. Technol. A 21, S194 (2003).

${ }^{5}$ S. Sreejith, P. Carol, P. Chithra, and A. Ajayaghosh, J. Mater. Chem. 18, 264 (2008).

${ }^{6}$ A. Ajayaghosh, Acc. Chem. Res. 38, 449 (2005).

${ }^{7}$ R. Petermann, M. Tian, S. Tatsuura, and M. Furuki, Dyes Pigm. 57, 43 (2003).

${ }^{8}$ M. C. Basheer, S. Alex, K. G. Thomas, C. H. Suresh, and S. Das, Tetrahedron 62, 605 (2006)

${ }^{9}$ D. Ramaiah, I. Eckert, K. T. Arun, L. Weidenfeller, and B. Epe, Photochem. Photobiol. 79, 99 (2004).

${ }^{10}$ M. C. Basheer, U. Santhosh, S. Alex, K. G. Thomas, C. H. Suresh, and S. Das, Tetrahedron 63, 1617 (2007).

${ }^{11}$ G. J. Ashwell, G. Jefferies, N. D. Rees, P. C. Williamson, G. S. Bahra, and C. R. Brown, Langmuir 14, 2850 (1998).

${ }^{12}$ S.-J. Chung, S. Zheng, T. Odani, L. Beverina, J. Fu, L. A. Padilha, A. Biesso, J. M. Hales, X. Zhan, K. Schmidt, A. Ye, E. Zojer, S. Barlow, D. J. Hagan, E. W. Van Stryland, Y. Yi, Z. Shuai, G. A. Pagani, J.-L. Bredas, J. W. Perry, and S. R. Marder, J. Am. Chem. Soc. 128, 14444 (2006).

${ }^{13}$ S. Webster, J. Fu, L. A. Padilha, O. V. Przhonska, D. J. Hagan, E. W. Van Stryland, M. V. Bondar, Y. L. Slominsky, and A. D. Kachkovski, Chem. Phys. 348, 143 (2008).

${ }^{14}$ D. Scherer, R. Dorfler, A. Feldner, T. Vogtmann, M. Schwoerer, U. Lawrentz, W. Grahn, and C. Lambert, Chem. Phys. 279, 179 (2002).

${ }^{15}$ K. Jyothish, M. Hariharan, and D. Ramaiah, Chem.-Eur. J. 13, 5944 (2007).

${ }^{16}$ A. J. McKerrow, E. Buncel, and P. M. Kazmaier, Can. J. Chem. 73, 1605 (1995).

${ }^{17}$ O. P. Dimitriev, A. P. Dimitriyeva, A. I. Tolmachev, and V. V. Kurdyukov, J. Phys. Chem. B 109, 4561 (2005)

${ }^{18}$ I. Cohanoschi, A. Barbot, K. D. Belfield, S. Yao, and F. E. Hernandez, J. Chem. Phys. 123, 231104 (2005).

${ }^{19}$ P. M. Kazmaier, G. K. Hamer, and R. A. Burt, Can. J. Chem. 68, 530 (1990).

${ }^{20}$ J. Lakowicz, Principles of Fluorescence Spectroscopy (Kluwer Academic, New York, 1999).

${ }^{21}$ M. Sheik-Bahae, A. Said, T. Wei, D. Hagan, and E. Van Stryland, IEEE J. Quantum Electron. 26, 760 (1990).

${ }^{22}$ L. De Boni, C. Toro, and F. E. Hernandez, Opt. Express 16, 957 (2008).

${ }^{23}$ R. E. Stratmann, G. E. Scuseria, and M. J. Frisch, J. Chem. Phys. 109, 8218 (1998)

${ }^{24}$ M. Cossi, V. Barone, B. Mennucci, and J. Tomasi, Chem. Phys. Lett. 286, 253 (1998).

${ }^{25}$ M. J. Frisch, G. W. Trucks, H. B. Schlegel et al., GAUSSIAN 03, Revision E.01, Gaussian, Inc., Wallingford, CT, 2004.

${ }^{26}$ A. D. Becke, J. Chem. Phys. 98, 5648 (1993).

${ }^{27}$ C. Lee, W. Yang, and R. G. Parr, Phys. Rev. B 37, 785 (1988).

${ }^{28}$ L. Tong and P. Bixian, Dyes Pigm. 39, 201 (1998).

${ }^{29}$ C. W. Dirk, W. C. Herndon, F. Cervantes-Lee, H. Selnau, S. Martinez, P. Kalamegham, A. Tan, G. Campos, M. Velez, J. Zyss, I. Ledoux, and L.-T. Cheng, J. Am. Chem. Soc. 117, 2214 (1995).

${ }^{30}$ C. A. T. Laia and S. M. B. Costa, Chem. Phys. Lett. 285, 385 (1998).

${ }^{31}$ X.-H. Li, B.-W. Zhang, and Y. Cao, Dyes Pigm. 45, 209 (2000).

${ }^{32}$ D. P. Craig and T. Thirunamachandran, Molecular Quantum Electrodynamics (Dover, Mineola, 1998).

${ }^{33}$ K. Ohta and K. Kamada, J. Chem. Phys. 124, 124303 (2006).

${ }^{34}$ P. Salek, O. Vahtras, T. Helgaker, and H. Agren, J. Chem. Phys. 117, 9630 (2002).

${ }^{35}$ S. Tretiak and V. Chernyak, J. Chem. Phys. 119, 8809 (2003).

${ }^{36}$ I. A. Mikhailov, S. Tafur, and A. E. Masunov, Phys. Rev. A 77, 012510 (2008).

${ }^{37}$ W. G. Han, T. Q. Liu, F. Himo, A. Toutchkine, D. Bashford, K. M. Hahn, and L. Noodleman, ChemPhysChem 4, 1084 (2003).

${ }^{38}$ F. Momicchioli, A. S. Tatikolov, D. Vanossi, and G. Ponterini, Photochem. Photobiol. Sci. 3, 396 (2004).

${ }^{39}$ D. Jacquemin, E. A. Perpete, G. E. Scuseria, I. Ciofini, and C. Adamo, J. Chem. Theory Comput. 4, 123 (2008).

${ }^{40}$ S. Ohira, I. Rudra, K. Schmidt, S. Barlow, S.-J. Chung, Q. Zhang, J. Matichak, S. R. Marder, and J.-L. Bredas, Chem.-Eur. J. 14, 11082 (2008).

${ }^{41}$ J. Bernstein and E. Goldstein, Mol. Cryst. Liq. Cryst. 164, 213 (1988). 